\title{
Aplicación de la Planeación por Escenarios en Unidades Académicas: Caso Programa de Administración Industrial
}

\section{Application of scenario planning in academic units: Industrial Management Program Case}

\author{
jvergaras@unicartagena.edu.co, Magister en Administración, profesor de tiempo completo del programa de Administración Industrial, \\ Universidad de Cartagena, Grupo de Investigación Métodos Cuantitativos de Gestión, Cartagena, Colombia
}

Juan Carlos Vergara-Schmalbach ${ }^{1}$

tfontalvoh@unicartagena.edu.co, Magister en Administración, profesor de tiempo completo del programa de Administración Industrial, Universidad de Cartagena, Grupo de Investigación Calidad y productividad organizacional Integral, Cartagena, Colombia

\section{Tomás Fontalvo Herrera ${ }^{2}$}

\begin{abstract}
jmorelosg@unicartagena.edu.co, Magister en Administración, profesor de tiempo completo del programa de Administración Industrial, Universidad de Cartagena, Grupo de Investigación Calidad y productividad organizacional Integral, Cartagena, Colombia
\end{abstract}

\section{José Morelos Gómez ${ }^{3}$}

Recibido: Marzo 14 de 2012

Aceptado: Abril 23 de 2012

\section{RESUMEN}

El programa de Administración Industrial de la Universidad de Cartagena, se ha caracterizado por su trayectoria académica y el desarrollo de avances en materia de investigación en todo el Departamento de Bolívar. A pesar de disponer de una documentación confiable de sus desarrollos, hasta el momento no se había aventurado en plantear estrategias con vigencia más allá de los 4 años. Estos nuevos retos fueron analizados aplicando el método de Planeación por Escenarios, con una secuencia de 10 pasos lineales, considerando métodos mixtos( Muskat, Blackman y Muskay, 2012).En esta Investigación descriptiva-propositiva, se emplearon herramientas tanto cuantitativas en la proyección de las variables, como cualitativas, en la valoración subjetiva de las probabilidades y el tratamiento de las variables incierta , cuyos resultados se evidencia en este artículo.. Los escenarios propuestos (validados por un panel de expertos) conducen a plantear estrategias (con límite de tiempo de 15 años) en torno a la cualificación de los docentes, al desarrollo de proyectos conjuntos con el sector privado y el diseño de un portafolio ampliado de cursos y seminarios, la oferta de postgrados propios, el fortalecimiento de los grupos de investigación y redes de investigación.

Palabras clave: Planeación por escenarios, toma de decisiones, unidades académicas, programas de pregrado.

\begin{abstract}
The Industrial Management program at the University of Cartagena, was characterized by his academic and development advances in research throughout the Department of Bolivar. Despite having a reliable documentation of their developments, so far had not ventured to raise effective strategies beyond the four years. These new challenges were analyzed using the method of scenario planning, the results can be seen in this article. The proposed scenarios (validated by a panel of experts) lead to propose strategies (with a time limit of 15 years) around the qualification of teachers, the development of joint projects with the private sector and the design of a broad portfolio of courses and seminars, offering postgraduate own, strengthening research groups and research networks.
\end{abstract}

Keywords: Scenario planning, decision making, academic units, undergraduate programs. 


\section{Introducción}

Con el propósito de conocer o anticiparse a los eventos que deparará el futuro, se han desarrollado diversas herramientas o métodos que encajan en modelos cuantitativos y cualitativos. Dichas herramientas por lo general tienen en cuenta: comportamientos o datos históricos, el conocimiento de expertos, técnicas matemáticas, estudios de mercado y/o análisis de tendencias. El límite está dado por el horizonte de tiempo, el conocimiento de la problemática e intereses de la organización, y la calidad de los datos históricos.

Los métodos cuantitativos (proyecciones, regresiones y tendencias) son peculiares cuando se trabaja con datos históricos, y horizontes de tiempo que no superan el corto y mediano plazo. Mientras mayor sea el periodo de tiempo considerado, existirá una mayor posibilidad de error en las proyecciones realizadas (la incertidumbre se incrementa en el largo plazo). Estas limitantes han originado, por parte de académicos y asesores empresariales, diversos métodos alternativos. La planeación por escenarios, es tal vez la herramienta más completa e integral empleada no solo para predecir, sino también para construir posibles futuros ideales (teniendo en cuenta las condiciones del entorno).

El presente artículo, surge por el interés (por parte de los autores) en aplicar el método de planeación por escenarios, en unidades académicas o programas de pregrado que tienen que construir o idealizar estrategias en el largo plazo. Con este propósito, se desarrolló un caso completo de prospectiva (basado en una adaptación del método SEARCH) para el programa de Administración Industrial de la Universidad de Cartagena.

\section{Revisión de la Literatura}

Para Wack (1985), el limitado conocimiento en implementar la planeación por escenarios, sugiere su escaso desarrollo y aplicabilidad en muchas organizaciones.

Se entiende como escenario a la descripción de un futuro potencial o posible, incluyendo el detalle de cómo llegar a ella (Godet, 2006; Porter, 1998), que explora el efecto conjunto de varios eventos (Schoemaker, 1995). Nicol (2005) en sus tesis doctoral (en filosofía) titulada "Scenario Planning as an Organisational Change Agent" realiza un análisis de distintos conceptos provenientes de 20 autores reconocidos en el mundo académico, concluyendo que los escenarios proveen de unos marcos o restricciones para analizar el futuro, limitando el número posible de futuros a ser considerados.

En este sentido, la planeación por escenarios se resume en poder anticiparse al futuro en el largo plazo (Comelius Van de Putte y Romani, 2005) entendiéndose como un aspecto crítico para el éxito de una organización, con el valor agregado a diferencia de los métodos tradicionales de pronóstico al considerar diferentes realidades y alternativas. La planeación por escenarios se consi- dera como parte de la planeación estratégica, relacionada con las herramientas y tecnologías para manejar la incertidumbre sobre el futuro ( Jay Ogilvy y Schwartz, 1998; Godet,2000; Lindgren y Bandhold;2003). Los escenarios por si solos no son una estrategia (Jay Ogilvy y Schwartz, 1996)

El diseño de los escenarios se sustentan en gran medida, en los aportes ofrecidos en el campo de la teorización por Dubin, Lynham y Van de Ven ( Chermack, 2005), siendo el de mayor influencia el método propuesto por Dubin definiendo una secuencia de ocho pasos que parte de la identificación de la unidad de estudio hasta la construcción de las hipótesis y pruebas de las mismas (Storberg-Walker, 2003).

En la literatura se pueden encontrar diferentes métodos para diseñar escenarios que combinan distintas herramientas (cualitativas y cuantitativas) (Postma y Liebl, 2005, ) coincidiendo en aportar ideas para enfrentar y aprovechar de una mejor forma las amenazas y oportunidades que depara el futuro.

Para la planeación por escenarios se han desarrollado diversos métodos, herramientas y metodologías (Anheier y Katz, 2009), catalogadas en tres grandes escuelas: la lógica e intuitiva, la prospectiva y de tendencia probabilística ( Bradfiel, Wright, Burt, Caims, y Van Der Heijden, 2005).

El primero en elaborar una metodología procedimental basada en una serie de pasos bien estructurados fue Peter Wack (1985), donde admite métodos cualitativos - basado en la concepción de Kahn - y cuantitativos. Las metodologías para la planeación por escenarios fueron evolucionando, integrando la toma de decisiones y estrategias para aprovechar mejor los escenarios creados. Dentro la variedad de métodos se resalta el propuesto por Peter Schwartz (1991) del cual dependerán otros modelos posteriores, quien define una metodología robusta de ocho pasos (List, 2007)

En 1992, la escuela prospectiva representada por Michael Godet, propone que los escenarios pueden servir no solo para predecir un futuro, sino como orientación para construir un futuro idealizado ( Godet y Roubelat, 1996)), integrándola como herramienta en los estudios prospectivos estratégicos ( Godet, 2000)

En las universidades, el factor determinante del futuro de las mismas está dada por la incertidumbre ( Munck y Mc Connell, 2009) Los escenarios por si mismos no resuelven el problema en las unidades académicas, solo proveerán de información sobre oportunidades y retos en el futuro, que podrían materializarse en acciones (Ogilvy, 1993).

Un plan estratégico o plan de desarrollo, documentos muy comunes en las instituciones educativas, deberían ser considerados como productos físicos, provenientes de un ejercicio de planeación por escenarios (Chang, 2008). Esto supondría elevar el horizonte de tiempo de las estrategias al largo plazo, que en la mayoría de los planes de colegios y universidades, apuntan a 
analizar las condiciones del entorno y evaluar las operaciones existentes (Mcintyre, 2011).

\section{Metodología}

El presente artículo, está basado en el desarrollo y conclusión de una investigación descriptiva-propositiva, cuyo objetivo principal consistió en aplicar el método de planeación por escenarios (visionando un horizonte de tiempo de 15 años) para el programa de Administración Industrial de la Universidad de Cartagena en el nivel administrativo.

El método SEARCH (método considerado en este estudio) se convierte en una propuesta independiente, que utiliza elementos numéricos para medir el grado de incertidumbre sin llegar a depender enteramente de los métodos cuantitativos en la construcción de los escenarios ( Sapio,1995), ya estos métodos involucra un mayor número de pasos, fundamentado en una descripción del problema por medio de variables clasificadas en tres tipos: constantes, predecibles e inciertas ( Pagani, 2009). Descomponer la situación en una serie de variables es una propuesta que proviene de Kahn (2009)

Para Sapio (1995) las variables que se categorizan como constantes equivalen a las características del sector que se mantienen estables en el tiempo; las variables predecibles corresponden a los campos cuyos valores pueden ser pronosticados (o proyectados) y cuyo resultados poseen muy poca probabilidad de cambio en el futuro; Por último, las variables inciertas son aquellas cuyo comportamiento en el largo plazo no pueden ser predichos con total certeza, como los cambios políticos, sociales, tecnológicos y económicos.

Los escenarios se definen interrelacionando las variables que caracterizan el sistema a través del método de impactos cruzado, usado para combinar eventos futuros examinando su efecto potencial ( Bañuls y Turoff, 2011) y se componen de una parte determinística y otra probabilística. Tanto los subescenarios como los escenarios, fueron valorados y validados por un panel de expertos, cuyas pautas necesarias para la constitución de sus perfiles se muestra a continuación.

- El panel de experto debe estar conformado por un equipo humano interdisciplinario ( Weimer-Jehle, 2008)

- Los expertos se dividen en: expertos conocedores del problema, y en clientes y/o consumidores ( Lindgre y Bandhold, 2003) A pesar de que el concepto "experto" es ambiguo, su elección se sustenta en la capacidad de plantearse o encarar un futuro (Godet, 2000) o de juzgar posibles futuros ( Sapio,1995)

- Según Kahn (2009), un experto debe conocer muy bien del pasado y presente, sobre la situación que será proyectada o por lo menos una situación similar, incluyendo los detalles de su desarrollo. Además se requiere que el panel este conformado por personas que han trabajado por si mismos un poco dentro de los procesos en la situación de interés y de algún modo han imaginado la fusión de estos elementos de pensamiento en una especie de pintura sobre el futuro.

Tabla No. 1. Descripción de las fases

\begin{tabular}{|c|c|c|}
\hline FASE & ACTIVIDAD & DESCRIPCIÓN \\
\hline \multirow[t]{3}{*}{1} & 1. Definición del Sistema & Se define el problema (en detalle) que será abordado por la planeación por escenarios. \\
\hline & $\begin{array}{l}\text { 2. Descomposición del sistema en fac- } \\
\text { tores y variables clave }\end{array}$ & $\begin{array}{l}\text { Se determinan } 3 \text { factores (o fuerzas de cambio) generales y las variables que caracterizan el } \\
\text { sistema, clasificándolas en constantes, predecibles e inciertas. }\end{array}$ \\
\hline & 3. Formulación de los subescenarios & $\begin{array}{l}\text { Una vez proyectadas las variables predecibles, enlazadas con las constantes y variables inciertas, } \\
\text { se construyen los subescenarios por cada factor clave. }\end{array}$ \\
\hline \multirow[t]{2}{*}{2} & 4. Selección del panel de expertos & $\begin{array}{l}\text { Se definen los perfiles del panel de expertos que participará en la valoración de los subescenarios } \\
\text { y validación de los escenarios. }\end{array}$ \\
\hline & $\begin{array}{l}\text { 5. Evaluación de los subescenarios por } \\
\text { el panel de experto }\end{array}$ & $\begin{array}{l}\text { Cada miembro del panel de experto valora subjetivamente cada subescenario de acuerdo a } \\
\text { una escala ordinal. }\end{array}$ \\
\hline \multirow[t]{3}{*}{3} & $\begin{array}{l}\text { 6. Selección de los subescenarios más } \\
\text { probables }\end{array}$ & $\begin{array}{l}\text { Se seleccionan tres subescenarios por cada factor, teniendo en cuenta aquellos que presentan } \\
\text { mayor probabilidad de ocurrencia. }\end{array}$ \\
\hline & $\begin{array}{l}\text { 7. Valoración de la compatibilidad entre } \\
\text { pares de escenarios }\end{array}$ & Equivale a una valoración numérica de acuerdo a una escala nominal de impacto. \\
\hline & $\begin{array}{l}\text { 8. Análisis de impacto cruzado y cálculo } \\
\text { de probabilidades }\end{array}$ & $\begin{array}{l}\text { Se evalúan el impacto de los escenarios (o conjunto de subescenarios) aplicando el método de } \\
\text { impacto cruzado. Se calculan las probabilidades para cada escenario. }\end{array}$ \\
\hline \multirow[t]{2}{*}{4} & 9. Redacción de escenarios & $\begin{array}{l}\text { Se describe el contenido de los escenarios, enlazando los subescenarios compatibles, incluyendo } \\
\text { los indicadores de alerta y esfuerzos requeridos. }\end{array}$ \\
\hline & 10. Validación de los subescenarios & Se realiza una valoración final de cada escenario por el panel de experto. \\
\hline
\end{tabular}

Fuente: Elaborado por los autores de acuerdo al método SEARCH de Sapio (1995). 
Manteniendo las bases del método SEARCH, se estableció una secuencia de 10 pasos lineales, considerando métodos mixtos (Muskat, Blackman y Muskay) al emplear herramientas tanto cuantitativas (en la proyección de las variables) como cualitativas (en la valoración subjetiva de las probabilidades y el tratamiento de las variables inciertas). En la Tabla 1, se puede observar en detalle la metodología empleada.

\section{Desarrollo del caso}

El programa de Administración Industrial de la Universidad de Cartagena es una unidad académica adscrita a la Facultad de Ciencias Económicas, cuyos procesos misionales (docencia, investigación y proyección) corresponden a los lineamientos institucionales.

El programa académico asume la misión de formar profesionales comprometidos con el desarrollo industrial de la Costa Caribe en particular y del país en general, mediante una fundamentación sólida en la administración de la actividad industrial como unidad compleja y la aplicación de los avances tecnológicos que respondan a los requerimientos del entorno industrial y empresarial.

A pesar de que el programa cuenta con una plan de desarrollo (con vigencia de 4 años), un plan de autoevaluación (con vigencia de 1 a 2 años) y un proyecto educativo vigente, hasta el momento no había realizado un ejercicio propio de planeación a largo plazo que permita dirigir los esfuerzos en los planes operativos de corto y mediano plazo, justificando en parte la realización de un proyecto prospectivo con horizonte de tiempo de 15 años.

- Fase 1- Identificación de los factores clave: La dirección del programa acordó como factores clave los procesos misionales de la institución: Docencia, investigación y proyección. De estos factores se relacionaron un total de 10 variables clasificadas en previsible, constantes e inciertas.
Las variables previsibles son variables cuantitativas cuyos datos históricos permitieron una proyección de sus contenidos. Dichas proyecciones fueron validadas con pruebas estadísticas y la dirección del programa (Tabla 2).

Por ejemplo, para la variable número de docentes se estimó que para el año 2026, el programa contará con 15 docentes de planta, valor estimado según los datos históricos recopilados y proyectados por medio de una regresión polinómica de orden 2. Estos datos se validaron de acuerdo al ideal de docentes establecido por el programa, el presupuesto de la universidad y el número de docentes registrados en otros programas similares (el valor óptimo de docentes de tiempo completo se fijó en 15).

Tabla No 2. Clasificación de las variables

\begin{tabular}{|c|c|c|}
\hline FACTOR & VARIABLES & $\begin{array}{c}\text { TIPO DE } \\
\text { VARIABLES }\end{array}$ \\
\hline DOCENCIA & Cualificación de docentes & Previsible \\
\hline & Número de Docentes & Previsible \\
\hline & Estudiantes & Constante \\
\hline INVESTIGACIÓN & $\begin{array}{c}\text { Egresados } \\
\text { Productos resultados de la } \\
\text { investigación }\end{array}$ & Previsible \\
\hline & $\begin{array}{c}\text { Grupos de investigación y } \\
\text { semilleros }\end{array}$ & Previsible \\
\hline PROYECCIÓN & Trabajos de grado & Previsible \\
\hline & $\begin{array}{c}\text { Trabajos de grado con impacto } \\
\text { social }\end{array}$ & Previsible \\
\hline & Programas sociales & Constante \\
\hline & Seminarios & Constante \\
\hline
\end{tabular}

Fuente: Elaborado por los autores

Tabla No $\mathbf{N}^{\circ}$. Cálculos de las regresiones del Sub-escenario A1

\begin{tabular}{|c|c|c|c|c|c|c|c|}
\hline \multirow[t]{2}{*}{ Tipo de regresión } & \multicolumn{4}{|c|}{ Constantes } & \multicolumn{2}{|c|}{$\begin{array}{c}\text { Prueba de Hipótesis sobre } \\
\text { la correlación }\end{array}$} & \multirow[t]{2}{*}{ FUNCIÓN } \\
\hline & $\mathbf{R}$ & $\mathbf{A}$ & B & C & t calculado & t crítico $( \pm)$ & \\
\hline Lineal & 0,970412 & $-1265,9$ & 0,6333 & - & 10,637 & 2,306 & $y=A+B X$ \\
\hline Potencial & 0,970567 & 253,71 & 0 & - & 7,404 & 2,306 & $Y=B X^{A}$ \\
\hline Exponencial & 0,971648 & 3 & $-0,126$ & - & 10,654 & 2,306 & $Y=A e^{B X}$ \\
\hline Logarítmica & 0,971648 & 1271 & -9660 & - & 5,646 & 2,306 & $Y=B \ln X-A$ \\
\hline Polinómica de Orden 2 & 0,973345 & 0,021 & 0,4169 & 2,4524 & 11,227 & 2,306 & $Y=A x^{2}+B X+C$ \\
\hline
\end{tabular}

Fuente: Elaborado por los autores 
La selección de la regresión polinómica de orden 2 se justificó por presentar una mayor correlación ( $r$ ) comparado con las correlaciones para las funciones lineal, potencial, exponencial y logarítmica. Los datos se verificaron mediante una prueba de bondad de ajuste para el valor de $r$ (ver Tabla 3) bajo las siguientes hipótesis.

\section{$H_{0}$ : Las variables $N O$ tienen una relación significativa \\ $H_{1}$ : Las variables SI tienen una relación significativa}

Los resultados -calculados con la ayuda del software Basic Forecasting versión 3.0 (Vergara-Schmalbach y Quesada, 2010)- de la prueba indican que en todos los casos se puede demostrar que existe una relación entre las variables año y número de docentes (los valores de están por fuera del rango ). En la Tabla 4 se muestran los resultados de la proyección.

Tabla No 4. Proyección del número de docentes de tiempo completo en el programa

\begin{tabular}{|c|c|}
\hline Año & $\begin{array}{l}\text { Número Docentes } \\
\text { (Acumulado) }\end{array}$ \\
\hline 2003 & 3 \\
\hline 2004 & 3 \\
\hline 2005 & 4 \\
\hline 2006 & 5 \\
\hline 2007 & 5 \\
\hline 2008 & 5 \\
\hline 2009 & 7 \\
\hline 2010 & 7 \\
\hline 2011 & 8 \\
\hline 2012 & 9 \\
\hline 2013 & 9 \\
\hline 2014 & 9 \\
\hline 2015 & 9 \\
\hline 2016 & 10 \\
\hline 1017 & 11 \\
\hline 2018 & 12 \\
\hline 2019 & 14 \\
\hline 2020 & 15 \\
\hline 2021 & 15 \\
\hline 2022 & 15 \\
\hline 2023 & 15 \\
\hline 2024 & 15 \\
\hline 2025 & 15 \\
\hline 2026 & 15 \\
\hline
\end{tabular}

Fuente: Elaborado por los autores
A partir de la interrelación de las variables se obtuvieron 12 subescenarios (4 por cada factor) como se muestran en la Tabla 5.

\section{Tabla No 5 . Listado de subescenarios}

\begin{tabular}{|l|l|}
\hline \multicolumn{1}{|c|}{ Nombre } & \multirow{2}{*}{ Factor Clave } \\
\cline { 1 - 1 } S1. Cualificación Docente & \multirow{2}{*}{ Docencia } \\
\cline { 1 - 1 } S2. Oferta académica & \\
\cline { 1 - 1 } S3. Oferta de programas de postgrado & \\
\cline { 1 - 1 } S4. Deserción estudiantil & \\
\cline { 1 - 1 } S5. Productividad académica & \\
\cline { 1 - 1 } S6. Posicionamiento de los grupos de investigación & \\
\cline { 1 - 1 } S7. Internacionalización de los grupos & \multirow{2}{*}{ Proyección } \\
\cline { 1 - 1 } S8. Relación empresa/programa & \\
\cline { 1 - 1 } S9. Proyectos de grado con énfasis en el impacto social & \\
\cline { 1 - 1 } S10. Oferta de cursosy seminariosa empresas/egresados & \\
\cline { 1 - 1 } S11. Desarrollo programas sociales & \\
\cline { 1 - 1 } S12.Seminarios a la comunidad estudiantil & \\
\cline { 1 - 2 } &
\end{tabular}

Fuente: Elaborado por los autores

- Fase 2 - Valoración de los subescenarios: Las personas que conformaron el panel de expertos (35 en total) se perfilaron por tener suficiente conocimiento acerca del programa, referente a su desempeño, sus proyectos y capacidades. El panel fue integrado por el decano y el vice-decano de la Facultad de Ciencias Económicas de la Universidad de Cartagena, el director del programa de Administración Industrial, docentes de planta del programa, el director del instituto de políticas públicas de la universidad, estudiantes que hacen parte de los semilleros y grupos de investigación y egresados del programa vinculados al sector empresarial. La valoración de los subescenarios se muestra en la Tabla 6 .

Tabla No 6. Promedio de valoración de los subescenarios

\begin{tabular}{|c|c|c|c|}
\hline $\begin{array}{c}\text { Sub- } \\
\text { escenarios }\end{array}$ & $\begin{array}{c}\text { Calificación } \\
\text { promedio }\end{array}$ & Probabilidad & Resultado \\
\hline S1 & 4,2 & $84,55 \%$ & PROBABLE \\
\hline S2 & 3,8 & $75,45 \%$ & POCO PROBABLE \\
\hline S3 & 4,1 & $82,73 \%$ & PROBABLE \\
\hline S4 & 4,1 & $81,82 \%$ & PROBABLE \\
\hline S5 & 4,0 & $80,00 \%$ & POCO PROBABLE \\
\hline S6 & 4,3 & $85,45 \%$ & PROBABLE \\
\hline S7 & 4,1 & $81,82 \%$ & PROBABLE \\
\hline S8 & 4,5 & $90,91 \%$ & PROBABLE \\
\hline S9 & 4,3 & $85,45 \%$ & PROBABLE \\
\hline S10 & 4,3 & $85,45 \%$ & PROBABLE \\
\hline S11 & 4,0 & $79,09 \%$ & POCO PROBABLE \\
\hline S12 & 4,0 & $80,91 \%$ & PROBABLE \\
\hline
\end{tabular}

Fuente: Elaborado por los autores 
Del total de sub-escenarios planteados el $75 \%$ fueron calificados por los expertos como probables y un $25 \%$ como poco probables. El sub-escenario con mayor probabilidad de ocurrencia es el S8 (90,91\%), seguido del S6, S9 Y S10 con una valoración de $85.45 \%$ para cada uno. Aquellos sub-escenarios que según el panel de expertos son pocos probable que sucediesen (sub-escenarios que tuvieron una valoración menor o igual de $80 \%$ ) en 15 años son el S2 (75,45\%), S5 (80,00\%) y el S11 (79,09\%).

- Fase 3 - Cálculo y formulación de los escenarios: Depurando los subescenarios (solo seleccionando aquellos calificados como probables), se generó un nuevo listado de eventos que serán tenidos en cuenta para el diseño de los escenarios (Tabla 7).

Tabla No 7. Listado de sub-escenarios seleccionados (3 por cada factor)

\begin{tabular}{|c|c|}
\hline \multirow{2}{*}{ Factor clave } & $\begin{array}{c}\text { Sub-escenario selec- } \\
\text { cionados }\end{array}$ \\
\hline \multirow{3}{*}{ Docencia } & $\mathrm{S} 1$ \\
\cline { 2 - 2 } & $\mathrm{S} 3$ \\
\cline { 2 - 2 } & $\mathrm{S} 4$ \\
\hline \multirow{3}{*}{ Investigación } & $\mathrm{S} 6$ \\
\cline { 2 - 2 } & $\mathrm{S} 7$ \\
\cline { 2 - 2 } & $\mathrm{S} 8$ \\
\hline \multirow{3}{*}{ Proyección } & $\mathrm{S} 9$ \\
\cline { 2 - 2 } & $\mathrm{S} 10$ \\
\cline { 2 - 2 } & $\mathrm{S} 12$ \\
\hline
\end{tabular}

Fuente: Elaborado por los autores

El número de combinaciones posibles entre los sub-escenarios probables de cada factor clave (se eligieron tres sub-escenarios de cada factor clave), determinando 27 escenarios en total (cálculo obtenido por la multiplicación de: 3 subescenarios del factor docencia, 3 subescenarios del factor investigación, y 3 subescenarios del factor proyección).

El panel de experto valoró las relaciones entre cada par de subescenarios teniendo en cuenta la escala de impacto formulada de la Tabla 8. Dicha valoración condujo al diseño de una matriz de impacto cruzado (Tabla 9).

El método o análisis de impacto cruzado es una poderosa herramienta cualitativa que evalúa un conjunto de eventos futuros (subescenarios) y examina su efecto potencial (Bañuls y Turoff: 2011). En el diseño de escenarios, este método se emplea para valorar la probabilidad de ocurrencia o impacto de un conjunto combinado de eventos (Fuchs et al., 2008).

Los datos se ingresaron en el software Scenario Wizard Basic 3 para obtener las combinaciones de subescenarios que causan mayor impacto al sistema. El software arrojó solo 4 combinaciones que presentaron una consistencia fuerte (Tabla 10). Dichas combinaciones seleccionadas dan pie a la construcción de los escenarios (Godet, 2000).

Tabla No 8. Escala Ordinal para la valoración causal de los subescenarios

\begin{tabular}{|c|l|}
\hline Intensidad del impacto & \multicolumn{1}{|c|}{ Descripción } \\
\hline+3 & Influencia directa promotora fuerte \\
\hline+2 & Influencia directa promotora moderada \\
\hline+1 & Influencia directa promotora débil \\
\hline 0 & Sin influencia \\
\hline-1 & Influencia directa restrictiva débil \\
\hline-2 & Influencia directa restrictiva moderada \\
\hline-3 & Influencia directa restrictiva fuerte \\
\hline
\end{tabular}

Fuente: Weimer-Jehle (2006)

Tabla No 9. Matriz de Impacto cruzado

\begin{tabular}{|c|c|c|c|c|c|c|c|c|c|}
\hline & S1 & S3 & S4 & S6 & S7 & S8 & S9 & S10 & S12 \\
\hline S1 & & & & 3 & 3 & 3 & 1 & 3 & 2 \\
\hline S3 & & & & 2 & 2 & 3 & 1 & 2 & 2 \\
\hline S4 & & & & 0 & 0 & 0 & 0 & 0 & 0 \\
\hline S6 & 3 & 2 & 0 & & & & 1 & 2 & 2 \\
\hline S7 & 3 & 2 & 1 & & & & 1 & 3 & 3 \\
\hline S8 & 2 & 2 & 0 & & & & 2 & 3 & 3 \\
\hline S9 & 2 & 1 & 0 & 1 & 1 & 2 & & & \\
\hline S10 & 3 & 3 & 0 & 3 & 3 & 3 & & & \\
\hline S12 & 3 & 1 & 0 & 2 & 3 & 2 & & & \\
\hline
\end{tabular}

Fuente: Elaborado por los autores

Los datos se ingresaron en el software Scenario Wizard Basic 3 para obtener las combinaciones de subescenarios que causan mayor impacto al sistema. El software arrojó solo 4 combinaciones que presentaron una consistencia fuerte (Tabla 10). Dichas combinaciones seleccionadas dan pie a la construcción de los escenarios (Godet, 2000).

Tabla No 10. Escenarios con calificaciones más altas de impacto

\begin{tabular}{|l|l|}
\hline Escenario No. $\mathbf{1}$ & Escenario No. $\mathbf{2}$ \\
\hline Puntaje de impacto total: $\mathbf{1 8}$ & Puntaje de impacto total: 17 \\
\hline A. DOCENCIA : $\mathbf{S 1}$ & A. DOCENCIA: S1 \\
\hline B. INVESTIGACIÓN: $\mathbf{S 7}$ & B. INVESTIGACIÓN: S6 \\
\hline C. PROYECCIÓN: S10 & C. PROYECCIÓN: S10 \\
\hline
\end{tabular}




\begin{tabular}{|l|l|}
\hline Escenario No. $\mathbf{3}$ & Escenario No. $\mathbf{4}$ \\
\hline Puntaje de impacto total: $\mathbf{1 7}$ & Puntaje de impacto total: $\mathbf{1 6}$ \\
\hline A. DOCENCIA : $\mathbf{S 1}$ & A. DOCENCIA : S3 \\
\hline B. INVESTIGACIÓN: S8 & B. INVESTIGACIÓN: S8 \\
\hline C. PROYECCIÓN: S10 & C. PROYECCIÓN: S10 \\
\hline
\end{tabular}

Fuente: Elaborado por los autores

En la Tabla siguiente se muestran los 4 escenarios con el cálculo respectivo de su probabilidad de ocurrencia en orden jerárquico (de mayor a menor probabilidad).

Tabla No 11. Escenarios con mayores probabilidades de ocurrencia

\begin{tabular}{|c|c|c|}
\hline ESCENARIO & SUB-ESCENARIOS & PROBABILIDAD \\
\hline \multirow[t]{3}{*}{1} & S1 & $84,55 \%$ \\
\hline & S8 & $90,91 \%$ \\
\hline & S10 & $85,45 \%$ \\
\hline $\begin{array}{c}\text { PROBABILIDAD DE } \\
\text { OCURRENCIA }\end{array}$ & $65,68 \%$ & \\
\hline ESCENARIO & SUB-ESCENARIOS & PROBABILIDAD \\
\hline \multirow[t]{3}{*}{2} & S3 & $82,73 \%$ \\
\hline & S8 & $90,91 \%$ \\
\hline & S10 & $85,45 \%$ \\
\hline $\begin{array}{l}\text { PROBABILIDAD DE } \\
\text { OCURRENCIA }\end{array}$ & $64,72 \%$ & \\
\hline ESCENARIO & SUB-ESCENARIOS & PROBABILIDAD \\
\hline \multirow[t]{3}{*}{3} & S1 & $84,55 \%$ \\
\hline & S6 & $85,45 \%$ \\
\hline & S10 & $85,45 \%$ \\
\hline $\begin{array}{l}\text { PROBABILIDAD DE } \\
\text { OCURRENCIA }\end{array}$ & $61.75 \%$ & \\
\hline ESCENARIO & SUB-ESCENARIOS & PROBABILIDAD \\
\hline \multirow[t]{3}{*}{4} & S1 & $84,55 \%$ \\
\hline & S7 & $81,82 \%$ \\
\hline & S10 & $85,45 \%$ \\
\hline $\begin{array}{l}\text { PROBABILIDAD DE } \\
\text { OCURRENCIA }\end{array}$ & $59,11 \%$ & \\
\hline
\end{tabular}

Fuente: Elaborado por los autores

\section{Resultados}

- Fase 4 - Redacción de los escenarios: Cumpliendo con esta última fase, a continuación se ilustra la redacción de los 4 escenarios del Programa de Administración Industrial para un horizonte de tiempo de 15 años.

ESCENARIO 1 - CUALIFICACIÓN DOCENTE, PROYECTOS CONJUNTOS CON EL SECTOR PRIVADO, Y PORTAFOLIO
DE CURSOS Y SEMINARIOS: El programa de Administración Industrial de la Universidad de Cartagena en el año 2026, contará con un total de 15 docentes cualificados como doctores en áreas afines o complementarias a la carrera.

El programa, realizará alianzas y proyectos con el sector privado a través de sus grupos de investigación, en donde se verán beneficiados ambas partes, al apoyar el sector privado con recursos financieros al programa para el fomento de una infraestructura científica, cooperación y financiamiento para la investigación, y a su vez el programa le ofrecerá la ayuda con proyectos investigativos. Esta fortaleza conducirá a la oferta de un portafolio de cursos y seminarios para empresas del sector empresarial.

\section{ESCENARIO 2 - OFERTA DE POSTGRADO, PROYECTOS CONJUNTOS CON EL SECTOR PRIVADO Y PORTAFOLIO DE CURSOS Y SEMINARIOS: El programa de Administración Industrial de la Universidad de Cartagena en 15 años, ofrecerá a la comunidad académica tres maestrías propias y un doctorado enfocado en áreas afines, a través del departamento de Postgrado de la Facultad de Ciencias Económicas, para mejorar la cualifica- ción de sus docentes y egresados.}

El programa, realizará alianzas y proyectos con el sector privado a través de sus grupos de investigación, en donde se verán beneficiados ambas partes, al apoyar el sector privado con recursos financieros al programa para el fomento de una infraestructura científica, cooperación y financiamiento para la investigación, y a su vez el programa le ofrecerá la ayuda con proyectos investigativos. Esta fortaleza conducirá a la oferta de un portafolio de cursos y seminarios para empresas del sector empresarial.

\section{ESCENARIO 3 - CUALIFICACIÓN DOCENTE, GRUPOS DE} INVESTIGACIÓN Y PORTAFOLIO DE CURSOS Y SEMINARIOS: El programa de Administración Industrial de la Universidad de Cartagena en el año 2026, contará con un total de 15 docentes cualificados como doctores en áreas afines o complementarias a la carrera, que liderarán los grupos de investigación adscritos al programa, gozando del reconocimiento del medio académico nacional e internacional (los grupos estarán reconocidos por Colciencias en su máxima categoría). Esta fortaleza conducirá a la oferta de un portafolio de cursos y seminarios para empresas del sector empresarial.

ESCENARIO 4 - CUALIFICACIÓN DOCENTES, REDES DE INVESTIGACIÓN Y PORTAFOLIO DE CURSOS Y SEMINARIOS:

El programa de Administración Industrial de la Universidad de Cartagena en el año 2026, contará con un total de 15 docentes cualificados como doctores en áreas afines o complementarias a la carrera. Los grupos de investigación se integrarán a redes internacionales de investigación, que permitirá el desarrollo de trabajos de investigación conjuntos con otros grupos de investigación en otros países. Esta fortaleza conducirá a la oferta de un portafolio de cursos y seminarios para empresas del sector empresarial. 


\section{Conclusiones}

En este artículo se demuestra la aplicabilidad del método de planeación por escenarios como coadyuvante en el proceso de toma de decisiones en unidades académicas.

Con el análisis de la historia y trayectoria del programa de Administración Industrial en el área de la docencia, Investigación y extensión, se identificaron las tendencias, constantes y elementos de incertidumbre que permitieron trazar los escenarios para un horizonte de tiempo de 15 años.

El método aquí planteado combinó las herramienta cualitativas (panel de expertos, método de impactos cruzados, análisis documental) y cuantitativas (proyecciones económicas y ambientales, probabilidades), promoviendo una visión holística de la situación.

La comunidad académica del programa deberá operacionalizar los escenarios anteriormente expuestos (en un plan estratégico), considerando en primera medida, el escenario de mayor probabilidad de ocurrencia (que combina los intereses y proyecciones de las directivas).

A través de este ejercicio se concluye, que la cualificación de los docentes (o incremento en su nivel de formación), es la estrategia reiterativa en la mayoría de los escenarios propuestos. Este impulso supondría un efecto directo en variables como la consolidación de los grupos y redes de investigación, de proyectos conjuntos con el sector privado y el desarrollo de un portafolio de cursos y seminarios.

Esta carta de navegación no es del todo estática (a pesar de considerar una pintura del estado del programa en el largo plazo), los esfuerzos también se deben concentrar en el seguimiento de los escenarios. Se propone entonces en el desarrollo de futuras investigaciones, diseñar una metodología o establecer los criterios para el control de los escenarios, integrándolos propiamente en el método de planeación por escenarios.

\section{Referencias}

Anheier, H. y Katz, H. (2009). Futures Research: Forecasting and Scenarios. Global Civil Society 2009: Poverty \& Activism (pp. 238-251). SAGE Publications Ltd.

Bañuls, V. A. y Turoff, M. (2011). Scenario construction via Delphi and cross-impact analysis. Technological Forecasting and Social Change, 78(9), 1579-1602. Elsevier Inc. doi:10.1016/j. techfore.2011.03.014.

Bradfield, R., Wright, G., Burt, G., Cairns, G. y Van Der Heijden, K. (2005). The origins and evolution of scenario techniques in long range business planning. Futures, 37(8), 795-812. doi:10.1016/j.futures.2005.01.003.
Chang, G.-chol. (2008). Strategic Planning in Education: Some Concepts and Methods. Direction in Educational Planning: Symposium (p. 14). Paris: International Institute for Educational Planning.

Chermack, T. J. (2005). Studying scenario planning: Theory, research suggestions, and hypotheses. Technological Forecasting and Social Change, 72(1), 59-73. doi:10.1016/j.techfore.2003.11.003.

Cornelius, P., Van de Putte, A. y Romani, M. (2005). Three Decades of Scenario Planning in Shell. California Management Review, 48(1), 92-109.

Fuchs, B. G., Fahl, U., Pyka, A., Staber, U., Voegele, S. y Weimerjehle, W. (2008). Department of Economics Generating Innovation Scenarios using the Cross-Impact Methodology. Bremen.

Godet, M. (2000). La caja de herramientas de la prospectiva estratégica. Paris.

Godet, M. (2006). Creating Futures: Scenario Planning as a Strategic Management Tool (2da ed., p. 349). London: Economica.

Godet, M. y Roubelat, F. (1996). Creating the future: The use and misuse of scenarios. Long Range Planning, 29(2), 164-171. doi:10.1016/0024-6301(96)00004-0.

Kahn, H. (2009). The Essential Herman Kahn. New York (p. 277). United Kingdom: Lexington Books.

Lindgren, M. y Bandhold, H. (2003). Scenario Planning: The link between future and strategy (p. 193). New York: Palgrave Macmillan.

List, D. (2007). From scenario planning to scenario network mapping. NZSSES 2007 Conference Proceedings and Presentations (p. 6). New Zealand: NZSSES - New Zealand Society for Sustainability Engineering and Science.

Mcintyre, C. (2011). Planning for a College's Future: Community Focus Groups, Scenarios, and Simulations. World Future Review, Fall(1), 57-65.

Munck, R. y McConnell, G. (2009). University Strategic Planning and the Foresight/Futures Approach: An Irish Case Study. Planning for Higher education, 4(1), 31-41.

Muskat, M., Blackman, D. y Muskat, B. (2012). Mixed Methods : Combining Expert Interviews , Cross- Impact Analysis and Scenario Development. The Electronic Journal of Business Research Methods, 10(1), 9-21.

Nicol, P. (2005). Scenaro Planning as an Organisational Change Agent. Curtin University Technology. 
Ogilvy, Jay y Schwartz, P. (1998). Plotting Your Scenarios (p. 20). Emeryville: GNB.

Ogilvy, James. (1993). Three Scenarios for Higher Education: The California Case. The Nea Higher Education Journal, 9(1), 25-67.

Pagani, M. (2009). Roadmapping $3 G$ mobile TV: Strategic thinking and scenario planning through repeated cross-impact handling. Technological Forecasting and Social Change, 76(3), 382-395. Elsevier Inc. doi:10.1016/j.techfore.2008.07.003.

Porter, M. E. (1998). Competitive Advantage: creating and sustaining superior performance (p. 592). New York: Free Press.

Postma, T. J. B. M. y Liebl, F. (2005). How to improve scenario analysis as a strategic management tool? Technological Forecasting and Social Change, 72(2), 161-173. doi:10.1016/j. techfore.2003.11.005.

Sapio, B. (1995). SEARCH (Scenario evaluation and analysis through repeated cross impact handling): a new method for scenario analysis with an application to the Videotel service in Italy. International Journal of Forecasting, 11(1), 113-131. doi:10.1016/0169-2070(94)02002-7.
Schoemaker, P. J. (1995). Scenario planning: a tool for strategic thinking. Sloan Management Review, 36(2), 25-40. doi:10.1016/0024-6301(95)91604-0.

Schwartz, P. (1991). The Art of the Long View (p. 272). New York: Currency Books/Doubleday.

Storberg-Walker, J. (2003). Comparison of the Dubin, Lynham, and Van de Ven Theory-Building Research Methods and Implications for HRD. Human Resource Development Review, 2(2), 211-222. doi:10.1177/1534484303002002007.

Vergara-Schmalbach, J. C y Quesada, V. (2010). Basic Forecasting. Cartagena: Universidad de Cartagena.

Wack, P. (1985). Scenarios: Uncharted Waters Ahead. Harvard Business Review, 63(5), 73-89.

Weimer-Jehle, W. (2006). Cross-impact balances: A systemtheoretical approach to cross-impact analysis. Technological Forecasting and Social Change, 73(4), 334-361. doi:10.1016/j. techfore.2005.06.005.

Weimer-Jehle, W. (2008). Cross-impact balances. Physica A: Statistical Mechanics and its Applications, 387(14), 3689-3700. doi:10.1016/j.physa.2008.02.006. 